\title{
Govind Swarup
}

\author{
Govind Swarup was a pioneer in the field of radio astronomy and the driving force behind two innovative Indian \\ radio telescopes. He contributed significantly to building up research institutions and promoting science education
} and training programs in the country.

W ith the passing of Govind Swarup on 7 September 2020 at the age of 91 , India lost a true scientific pioneer whose single-minded dedication and effort established the country as one of the leaders in radio astronomy.

Govind, as he was almost universally called by all who knew him, is perhaps best remembered as the person responsible for the construction of two large radio telescopes in India, the Ooty Radio Telescope (ORT) and the Giant Metrewave Radio Telescope (GMRT). Both telescopes were constructed on shoe-string budgets, but made significant contributions to our understanding of the Universe. That these unique facilities could be constructed is a testament to Govind's ability to come up with radically out-of-the-box designs, as well as to his courage and persistence in successfully taking on large engineering projects in an environment that provided scant support compared to what his contemporaries in the West took for granted.

Born in 1929 in pre-independence India, to a rich land-owning family, Govind Swarup was attracted to science from an early age. He obtained a masters degree from the University of Allahabad, after which he joined the National Physical Laboratory (NPL) in Delhi, where the then director K. S. Krishnan was trying to put together a group to work in the nascent field of radio astronomy. Unfortunately, this effort did not come to fruition. Govind moved on to work with well-known radio astronomy groups in Australia and the United States, and eventually did a Ph.D. at Stanford, working with Ron Bracewell. Pioneering work that he did during this period includes the discovery of solar ' $U$ ' bursts and the invention (along with $\mathrm{K}$. Yang) of the now widely used Swarup-Yang technique for providing phase stability for large radio arrays ${ }^{1}$. Following his Ph.D. he took up a position as assistant professor at Stanford in 1961, but the dream of building up radio astronomy in India remained alive. Along with contemporaries from the by then defunct radio astronomy group at NPL, he sent a proposal to set up a radio astronomy group to several Indian research institutions. The most encouraging response was from Homi Bhaba at the Tata

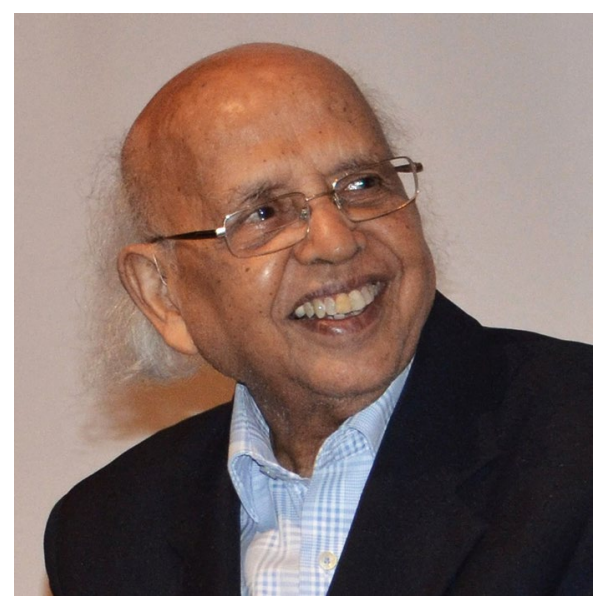

Govind Swarup (1929-2020). Credit: NCRA

Institute of Fundamental Research (TIFR) in Mumbai. Govind joined TIFR in 1963, and started the process of setting up a radio astronomy group.

After building a small but useful array near Mumbai for solar studies, Govind initiated an extremely ambitious project to build a large radio telescope, the idea being to use lunar occultations to look for evolution in the size of radio sources and thus distinguish between the competing cosmological models of the time. His ingenious idea was to construct a cylindrical telescope on a north-south hill slope parallel to the rotation axis of the earth. This would allow sources to be tracked by just rotating about a single axis. The large - $530 \mathrm{~m}$ long and $30 \mathrm{~m}$ wide - $\mathrm{ORT}^{2}$, built in the late 1960s following this design, played an important role in determining the evolution of the size of radio sources, as well as in studies of the Galactic ionized interstellar medium and space weather ${ }^{3-5}$. It also served as the training ground for generations of Indian radio astronomers. Indeed, to this day, Govind's students and their students form the bulk of the Indian radio astronomy community.

Govind's next big project was the GMRT, an array of 30 dishes, each $45 \mathrm{~m}$ in diameter, located near Pune ${ }^{6}$. Conceived in the mid-1980s, with construction starting in the early 1990s and becoming fully operational in early 2002, the GMRT was meant to fill an important global niche for a high-sensitivity low-frequency radio interferometer. Building such a large telescope at a modest cost was a significant challenge, particularly since at that time the country was going through a major financial crisis. In Govind's breakthrough design, which made optimum use of the fact that the GMRT is a low-frequency telescope, the parabolic reflecting surface was a stretched wire mesh pulled into the desired shape using suitably tensed wires. In conventional designs, developed for higher frequencies, the reflecting surface is made up of panels fixed onto a parabolic shaped backup structure. The result of his innovation was an extremely lightweight structure, with minimal wind loading, which could be constructed at a fraction of the cost of a conventional radio telescope. The project was innovative in many other ways, for example in using fibre-optic signal transmission. The GMRT, which has recently been significantly upgraded, remains one of the most sensitive metre wavelength radio interferometers in the world ${ }^{7}$.

Although Govind worked mainly in India, large collaborative international projects remained part of his vision for radio astronomy. He was one of the first to propose a large international radio telescope, with a collecting area several times larger than the GMRT, aimed at detecting hydrogen gas in distant galaxies. This idea, carried forward by others (including the National Centre for Radio Astrophysics, founded by Govind), has now taken shape as the international Square Kilometre Array project.

Govind's influence on science in India was much larger than just building radio telescopes. For him, science was always about training people and contributing to nation building. Govind never lost track of the importance of grooming the next generation of trained scientists, and many of the students attending the summer schools that he organized are now senior figures in the Indian astronomy scene. Later, he played an important role in starting the Joint Astronomy Programme, a shared doctoral programme run by several research institutes in Bangalore. 
This programme is still going strong, and has produced generations of outstanding astronomers. Concerns about the lack of adequately trained manpower in science and engineering remained important to Govind even after he had retired from TIFR. He was one of the main drivers behind a proposal to establish new institutes for undergraduate training in science and engineering. This concept eventually metamorphosed into the Indian Institutes of Science Education and Research (IISERs). The seven IISERs that have been established by the government of India have already made their mark in the training of the next generation of Indian scientists across a whole range of disciplines.

Over his lifetime Govind won many recognitions and awards, including the Padma Shri (one of India's highest civilian awards), the Homi Bhabha Lifetime
Achievement Award from the prime minister of India, the John Howard Dellinger Gold Medal of the International Union of Radio Sciences, and the Grote Reber Medal. He was also elected fellow of a number of science academies, including the Royal Society.

Although Govind was easily one of the most accomplished scientists in India, he remained down to earth, and always eager to communicate with scientists, engineers and students. He was a meticulous mentor, quick to identify and encourage talent, maintaining contact and encouraging his extended family of protégés long after their formal association with him had ended. To the end he retained his curiosity about all things scientific as well as his characteristic, infectious enthusiasm. His absence will be sorely felt not just by the scientific community, but by all those who had the good fortune to interact with him.

\section{Jayaram N. Chengalur ${ }^{凶}$ and}

Yashwant Gupta (D)

National Centre for Radio Astrophysics, Pune

University Campus, Pune, India. ${ }_{e}$-mail:

chengalur@ncra.tifr.res.in; ygupta@ncra.tifr.res.in

Published online: 9 November 2020

https://doi.org/10.1038/s41550-020-01259-y

References

1. Swarup, G. \& Yang, K. S. IEEE Trans. Antennas Propag. Ap-9, 75-81 (1961).

2. Swarup, G. et al. Nat. Phys. Sci. 230, 185-188 (1971).

3. Swarup, G. Mon. Not. R. Astron. Soc. 172, 501-512 (1975).

4. Anantharamaiah, K. R. J. Astrophys. Astron. 6, 177-201 (1985)

5. Manoharan, P. K. \& Ananthakrishnan, S. Mon. Not. R. Astron. Soc. 244, 691-695 (1990).

6. Swarup, G. et al. Current Sci. 60, 95-105 (1991).

7. Gupta, Y. et al. Current Sci. 113, 707-714 (2017). 